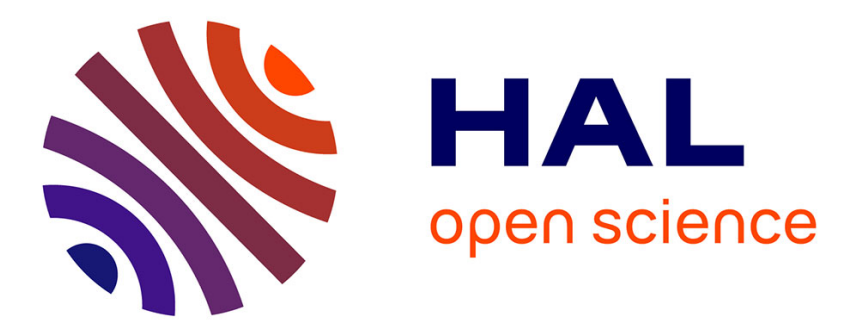

\title{
A Multi-Criteria Experimental Ranking of Distributed SPARQL Evaluators
}

Damien Graux, Louis Jachiet, Pierre Genevès, Nabil Layaïda

\section{To cite this version:}

Damien Graux, Louis Jachiet, Pierre Genevès, Nabil Layaïda. A Multi-Criteria Experimental Ranking of Distributed SPARQL Evaluators. Big Data 2018 - IEEE International Conference on Big Data, Dec 2018, Seattle, United States. pp.1-10. hal-01381781v2

\section{HAL Id: hal-01381781 \\ https://hal.inria.fr/hal-01381781v2}

Submitted on 22 Nov 2018

HAL is a multi-disciplinary open access archive for the deposit and dissemination of scientific research documents, whether they are published or not. The documents may come from teaching and research institutions in France or abroad, or from public or private research centers.
L'archive ouverte pluridisciplinaire HAL, est destinée au dépôt et à la diffusion de documents scientifiques de niveau recherche, publiés ou non, émanant des établissements d'enseignement et de recherche français ou étrangers, des laboratoires publics ou privés. 


\title{
A Multi-Criteria Experimental Ranking of Distributed SPARQL Evaluators
}

\author{
Damien Graux $^{\S \ddagger}$, Louis Jachiet ${ }^{\dagger \ddagger}$, Pierre Genevès ${ }^{\ddagger}$, Nabil Layaïda ${ }^{\ddagger}$ \\ ${ }^{\S}$ Enterprise Information Systems, Fraunhofer IAIS - Sankt Augustin, Germany \\ ${ }^{\dagger}$ Département Informatique ENS, PSL - Paris, France \\ $\ddagger$ Univ. Grenoble Alpes, CNRS, Inria, LIG - F-38000 Grenoble, France
}

\begin{abstract}
SPARQL is the standard language for querying RDF data. There exists a variety of SPARQL query evaluation systems implementing different architectures for the distribution of data and computations. Differences in architectures coupled with specific optimizations, for e.g. preprocessing and indexing, make these systems incomparable from a purely theoretical perspective. This results in many implementations solving the SPARQL query evaluation problem while exhibiting very different behaviors, not all of them being adapted in any context. We provide a new perspective on distributed SPARQL evaluators, based on multi-criteria experimental rankings. Our suggested set of 5 features (namely velocity, immediacy, dynamicity, parsimony, and resiliency) provides a more comprehensive description of the behaviors of distributed evaluators when compared to traditional runtime performance metrics. We show how these features help in more accurately evaluating to which extent a given system is appropriate for a given use case. For this purpose, we systematically benchmarked a panel of 10 state-of-the-art implementations. We ranked them using a reading grid that helps in pinpointing the advantages and limitations of current technologies for the distributed evaluation of SPARQL queries.
\end{abstract}

Keywords-SPARQL, Distributed Evaluation, Benchmarking

\section{INTRODUCTION}

With the increasing availability of RDF [1] data, the $\mathrm{W} 3 \mathrm{C}$ standard SPARQL language [2] plays a role more important than ever for retrieving and manipulating data. Recent years have witnessed the intensive development of distributed SPARQL evaluators [3] with the purpose of improving the way SPARQL queries are executed on distributed platforms for more efficiency on large RDF datasets.

Two factors heavily contributed to offer a large design space for improving distributed query evaluators. First, the adoption of native data representations for preserving structure (propelled by the so-called "NoSQL" initiatives) offered opportunities for leveraging locality. Second, the seminal results on the MapReduce paradigm [4] triggered a rapid development of infrastructures offering primitives for distributing data and computations [5], [6]. As a result, the current landscape of SPARQL evaluators is very rich, encompassing native RDF systems (e.g. 4store [7]), extensions of relational DBMS (e.g. S2RDF [8]), extensions of NoSQL systems (e.g. CouchBaseRDF [9]). These systems leverage different representa-

Corresponding author: Damien Graux \{damien.graux @iais.fraunhofer.de

This research was partially supported by the ANR project CLEAR (ANR16-CE25-0010) and by the European Union's H2020 research and innovation action HOBBIT under the Grant Agreement number 688227. tions of RDF data for evaluating SPARQL queries, such as e.g. vertical partitioning [10] or key-value tables [11]. They also rely on different technologies for distributing subquery computations and for the placement and propagation of RDF triples: some come with their own distribution scheme (e.g. 4store [7]), others prefer distributed file systems such as HDFS [12] (e.g. RYA [11]), while yet others aim at taking advantage of higher-level frameworks such as PigLatin [6] or Apache Spark [5] (e.g. S2RDF [8]). Last but not least, many SPARQL evaluators implement optimizations targeting specific query shapes (e.g. CliqueSquare [13] that attempts to flatten execution plans for nested joins). This overall richness and variety in distributed SPARQL evaluation systems make it hard to have a clear global picture of the respective advantages and limitations of each system in practical terms.

Contribution. We provide a new perspective on distributed SPARQL evaluators, based on a multi-criteria ranking obtained through extensive experiments. Specifically, we propose a set of five principal features (namely velocity, immediacy, dynamicity, parsimony, and resiliency) which we use to rank evaluators. Each system exhibits a particular combination of these features. Similarly, the various requirements of practical use cases can also be decomposed in terms of these features.

Our suggested set of features provides a more comprehensive description of the behavior of a distributed evaluator when compared to traditional performance metrics. We show how it helps in more accurately evaluating to which extent a given system is appropriate for a given use case. For this purpose, we systematically benchmarked a panel of 10 state-of-the-art implementations. We ranked them using this reading grid to pinpoint the advantages and limitations of current SPARQL evaluation systems.

Outline. The rest of this paper is organized as follows. We first briefly describe the tested systems in Section II. In Section III, we introduce the methodology and the experimental protocol we used i.e. the datasets, the queries and the observed metrics. We then review in Section IV the experiences for each store. In Section V, we discuss the most appropriate systems based on the requirements of different features. Finally, we review related work in Section VI before concluding in Section VII.

\section{BENCHMARKED DATASTORES}

We first describe the systems used in our tests, focusing on their particularities for supporting RDF querying. We used 


\begin{tabular}{|c|c|c|c|c|c|}
\hline & Systems & Underlying Framework & Storage Back-End & Storage Layout & SPARQL Fragment \\
\hline \multirow{3}{*}{$\begin{array}{l}\text { Standalone } \\
\text { Datastores }\end{array}$} & 4store & - & Data Fragments & Indexes & SPARQL 1.0 \\
\hline & CumulusRDF & Cassandra & Key-Value store & 3 hash and sorted indexes & SPARQL 1.1 \\
\hline & CouchBaseRDF & CouchBase & Buckets & 3 views & Basic Graph Pattern \\
\hline \multirow{4}{*}{$\begin{array}{l}\text { HDFS-based } \\
\text { Datastores with } \\
\text { preprocessing }\end{array}$} & $\overline{\text { RYA }}$ & Accumulo & Key-Value store on HDFS & 3 sorted indexes & Basic Graph Pattern \\
\hline & SPARQLGX & Spark & Files on HDFS & Vertically Partitioned Files & Basic Graph Pattern \\
\hline & S2RDF & SparkSQL & Tables on HDFS & Extended Vertically Partitioned Files & Basic Graph Pattern \\
\hline & CliqueSquare & Hadoop & Files on HDFS & Indexes & Basic Graph Pattern \\
\hline \multirow{3}{*}{$\begin{array}{l}\text { HDFS-based Direct } \\
\text { Evaluators }\end{array}$} & PigSPARQL & PigLatin & Files on HDFS & N-Triples Files & SPARQL 1.0 \\
\hline & RDFHive & Hive & Relational store on HDFS & Three-column Table & Basic Graph Pattern \\
\hline & SDE & Spark & Files on HDFS & N-Triples Files & Basic Graph Pattern \\
\hline
\end{tabular}

TABLE I: Systems used in our tests.

several criteria in the selection of the SPARQL evaluators tested. First, we choose to focus on distributed evaluators so that we can consider datasets of more than 1 billion triples which is larger than the typical memory of a single node in a commodity cluster. Furthermore, we retained systems that support at least a minimal fragment of SPARQL composed of conjunctive queries and called the BGP fragment (further detailed in Section II-A). We focused on open-source systems. We wanted to include some widely used systems to have a well-known basis of comparison, as well as more recent research implementations. We also wanted our candidates to represent the variery and the richness of underlying frameworks, storage layouts, and techniques found - see e.g. taxonomies of [3] and [14] -, so that we can compare them on a common ground. We finally selected a panel of 10 candidate implementations, presented in Table I.

Table I also summarizes the characteristics of the systems we used in our tests. We split our panel of 10 implementations into subcategories. The first category, called standalone systems, gathers systems that distribute data using their own custom methods. In contrast, all the other systems use the well-known HDFS distributed file system [12] for this purpose. HDFS handles the distribution of data across the cluster and its replication. It is a tool included in the Apache Hadoop ${ }^{1}$ project which is a framework for distributed systems based on the MapReduce paradigm [4].

We further subdivide the HDFS-based systems into two categories: the preprocessing-based evaluators and the direct SPARQL evaluators. The first category requires some preprocessing whereas direct SPARQL evaluators use distributed data without preprocessing. We first summarize some required background on SPARQL and then further review the candidates of each category below.

\section{A. SPARQL Preliminaries}

The Resource Description Framework (RDF) is a language standardized by W3C to express structured information on the Web as graphs [1]. RDF data is structured in sentences, each one having a subject, a predicate and an object. SPARQL is the standard query language for retrieving and manipulating RDF data. It constitutes one key technology of the semantic web and has become very popular since it became an official w3C recommendation [2].

The SPARQL language has been extensively studied in the literature under the form of various fragments. In this study,

\footnotetext{
${ }^{1}$ http://hadoop.apache.org/
}

we focus on the Basic Graph Pattern (BGP) fragment which is composed of the set of conjunctive queries. The BGP fragment represents the core of the SPARQL language. Technically, conjunctive queries present a list of conditions on triples called triple patterns (TPS) each one describing required properties on the parts of an RDF sentence. The TP thus constitutes the basic building block of SPARQL queries for selecting the subset of triples where some subject, predicate or object match given values. See [2] for a more formal presentation of TPS and BGPS.

\section{B. Selected Datastores}

We now briefly introduce the selected evaluators:

1) 4store is a native RDF solution introduced in [7].

2) CumulusRDF [15] relies on Apache Cassandra.

3) CouchBaseRDF [9] uses CouchBase.

4) RYA [11] is a solution leveraging Apache Accumulo.

5) SPARQLGX [16] is based on Apache Spark.

6) S2RDF [8] uses SparkSQL.

7) CliqueSquare [13] is a native RDF solution.

8) PigSPARQL [17] compiles SPARQL to PigLatin.

9) RDFHive [16] uses tables with Apache Hive.

10) SDE [16] is a modification of SPARQLGX.

\section{Methodology For Experiments}

For studying how well the distribution techniques perform, we tested the 10 systems presented in Section II with queries from two popular benchmarks (LUBM and WatDiv), which we evaluated on several datasets of varying size. We precisely monitored the behavior of each system using several metrics encompassing e.g. total time spent, CPU and RAM usage, as well as network traffic. In this Section, we describe our experimental methodology in further details.

\section{A. Datasets and Queries}

As introduced in Section II-A, we focus here on the Basic Graph Pattern (BGP) fragment which is composed of the set of conjunctive queries. It is also the common fragment supported by all tested stores and thus provides a fair and common basis of comparison.

Also for a fair comparison of the systems introduced in Section II, we decided to rely on third-party benchmarks. The literature about benchmarks is also abundant (see e.g. [18] for a recent survey). For the purpose of this study, we selected benchmarks according to two conditions: (1) queries should focus on testing the BGP fragment and (2) the benchmark must 
be popular enough in order to allow for further comparisons with other related studies and empirical evaluations (such as [9] for instance). In this spirit, we retained the LUBM benchmark $^{2}$ [19] and the WatDiv benchmark ${ }^{3}$ [20].

LUBM is composed of two tools: a determinist parametric RDF triples generator and a set of fourteen queries. Similarly, WatDiv offers a determinist data generator which creates richer datasets than the LUBM one in the sens of the number of classes and predicates, in addition, it also comes with a query generator and a set of twenty query templates. We used several standard LUBM and WatDiv datasets with varying sizes to test the scalability of the compared RDF datastores. Table II presents the characteristics of datasets we used. We selected in particular these three ones because they are gradually RAMlimiting: the WatDiv1k dataset can be held in memory of one single VM, the Lubm1k dataset becomes too large and Lubm10k is larger than the whole available RAM of our cluster.

\begin{tabular}{|c|c|c|}
\hline Datasets & Number of Triples & Size \\
\hline WatDiv1k & 109 million & $15 \mathrm{~GB}$ \\
\hline Lubm1k & 134 million & $23 \mathrm{~GB}$ \\
\hline Lubm10k & 1.38 billion & $232 \mathrm{~GB}$ \\
\hline
\end{tabular}

TABLE II: Size of sample datasets.

We evaluated on these datasets the provided LUBM queries and generated the WatDiv queries according to the provided templates. LUBM queries (Q1-Q14) were made to represent real-world queries while remaining in the BGP fragment of SPARQL and with a small data complexity (the size of the answer for a query is always almost linear in the size of the dataset). In addition, in the LUBM query set, we notice that one query is challenging: Q2 since it involves large intermediate results and implies a complex join pattern called "triangular". WatDiv queries compared with LUBM ones involved more predicates and classes. Furthermore, WatDiv developers already group query templates according to four categories: linear queries (L1-L5), star queries (S1-S7), snowflake-shaped queries (F1-F5) and complex queries (C1-C3).

\section{B. Metrics}

During our tests we monitored each task by measuring not only time spent but a broader set of indicators:

1) Time (Seconds): simply measures the time taken by the system to complete a task.

2) Disk footprint (Bytes): measures the use of disks for a given dataset size including indices and any auxiliary data structures.

3) Disk activity (Bytes/second): measures at each instant the amount of bytes written on and read from the disks during processes.

4) Network traffic (Bytes/second): measures how much data is exchanged between nodes in the cluster.

5) $\quad C P U$ usage (percentage): measures how much the $\mathrm{CPU}$ is active during the computation.

6) RAM usage (Bytes): measures how much the RAM is used by the computation.

\footnotetext{
${ }^{2}$ http://swat.cse.lehigh.edu/projects/lubm/

${ }^{3}$ http://dsg.uwaterloo.ca/watdiv/
}

7) SWAP usage (Bytes): measures how much SWAP is used. Such a metric will be particularly measured when the system runs out of RAM and thus be often omitted.

\section{Cluster Setup}

Our experiments were conducted on a cluster composed of Virtual Machines (VMs) hosted on two servers. The first server has two processors Intel(R) Xeon(R) CPU E5-2620 cadenced at $2.10 \mathrm{GHz}, 96 \mathrm{GigaBytes}(\mathrm{GB})$ of RAM and hosts five VMs. The second server has two processors Intel(R) Xeon(R) CPU E5-2650 cadenced at $2.60 \mathrm{GHz}$ with $130 \mathrm{~GB}$ of RAM and hosts 6 VMs: 5 dedicated to the computation (like the $5 \mathrm{VM}$ of the first server) plus one special VM that orchestrates the computation. Each VM has dedicated 2 physical cores (thus 4 logical cores), 17 GB of RAM and 6 TeraBytes (TB) of disk. The network allows two VMs to communicate at 125 MegaBytes per Seconds (MB/s) but the total link between the two servers is limited at $110 \mathrm{MB} / \mathrm{s}$. The read and write speeds are $150 \mathrm{MB} / \mathrm{s}$ and $40 \mathrm{MB} / \mathrm{s}$ shared between the $\mathrm{VM}$ on the first server and $115 \mathrm{MB} / \mathrm{s}$ and $12 \mathrm{MB} / \mathrm{s}$ shared between the $\mathrm{VM}$ of the second server.

\section{Extensive Experimental Results}

We made our extensive experimental results openly available online ${ }^{4}$ with more detailed information. In particular, for reproducibily purposes, we wrote tutorials on how to install and configure the various tested evaluators and report all the versions of the systems we used. We also share measurements and graphs for all the considered metrics and for each node.

In the rest of the paper, we focus on summarizing and discussing the essence of the lessons that we learned from our experiments. In Section IV we report on the overall behavior of each system pushed to the limits during the tests. In Section V we further discuss and develop a comparative analysis guided by practical features that imply different requirements.

\section{OVERALl BehaVior OF Systems}

In this Section we report on the overall behavior of each tested systems for the three datasets presented in Table II, namely WatDiv1k, Lubm1k and Lubm10k. These datasets constitute appropriate yardsticks for studying how the tested systems behave when the dataset size grows, with the characteristics of the cluster used ( $c f$. Section III-C). Specifically, the WatDiv1k dataset can still be held in memory of one single VM, while the Lubm1k dataset becomes too large. Lubm10k is even larger than the whole available RAM of the cluster.

Figure 1a presents the times spent by each datastore for preprocessing the datasets ${ }^{5}$. Figure $1 \mathrm{~b}$ summarizes the problematic cases. Figures $1 \mathrm{c}, 1 \mathrm{~d} \& 1 \mathrm{e}$ respectively show the elapsed times for evaluating queries over WatDiv1k, Lubm1k and Lubm10k.

We further comment on the behavior of each system pushed to the limits below, and conclude this section with comparative and more general observations.

\footnotetext{
${ }^{4}$ http://tyrex.inria.fr/sparql-comparative/home.html

${ }^{5}$ Times reported for the HDFS-based systems do not include the times required to import the original files on the distributed file system.
} 


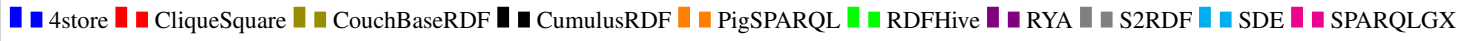

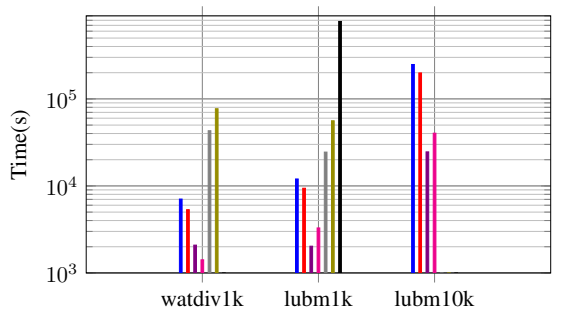

(a) Preprocessing Time.

\begin{tabular}{|r|c|c|c|}
\hline Evaluator & WatDiv1k & Lubm1k & Lubm10k \\
\hline CliqueSquare & F1,2,5 \& S2,3,5,6,7 Parser & $\varnothing$ & $\varnothing$ \\
\hline CouchBaseRDF & C3 Failure & Q2,14 Failure & Pre-processing Failure \\
\hline RDFHive & $\varnothing$ & Q2 Timeout & Q2 Timeout \\
\hline RYA & C2,3 Timeout & Q2 Timeout & Q2 Timeout \\
\hline S2RDF & $\varnothing$ & $\varnothing$ & Pre-processing Failure \\
\hline SDE & $\varnothing$ & Q2 Timeout & Q2 Timeout \\
\hline
\end{tabular}

(b) Failure Summary for problematic evaluators.
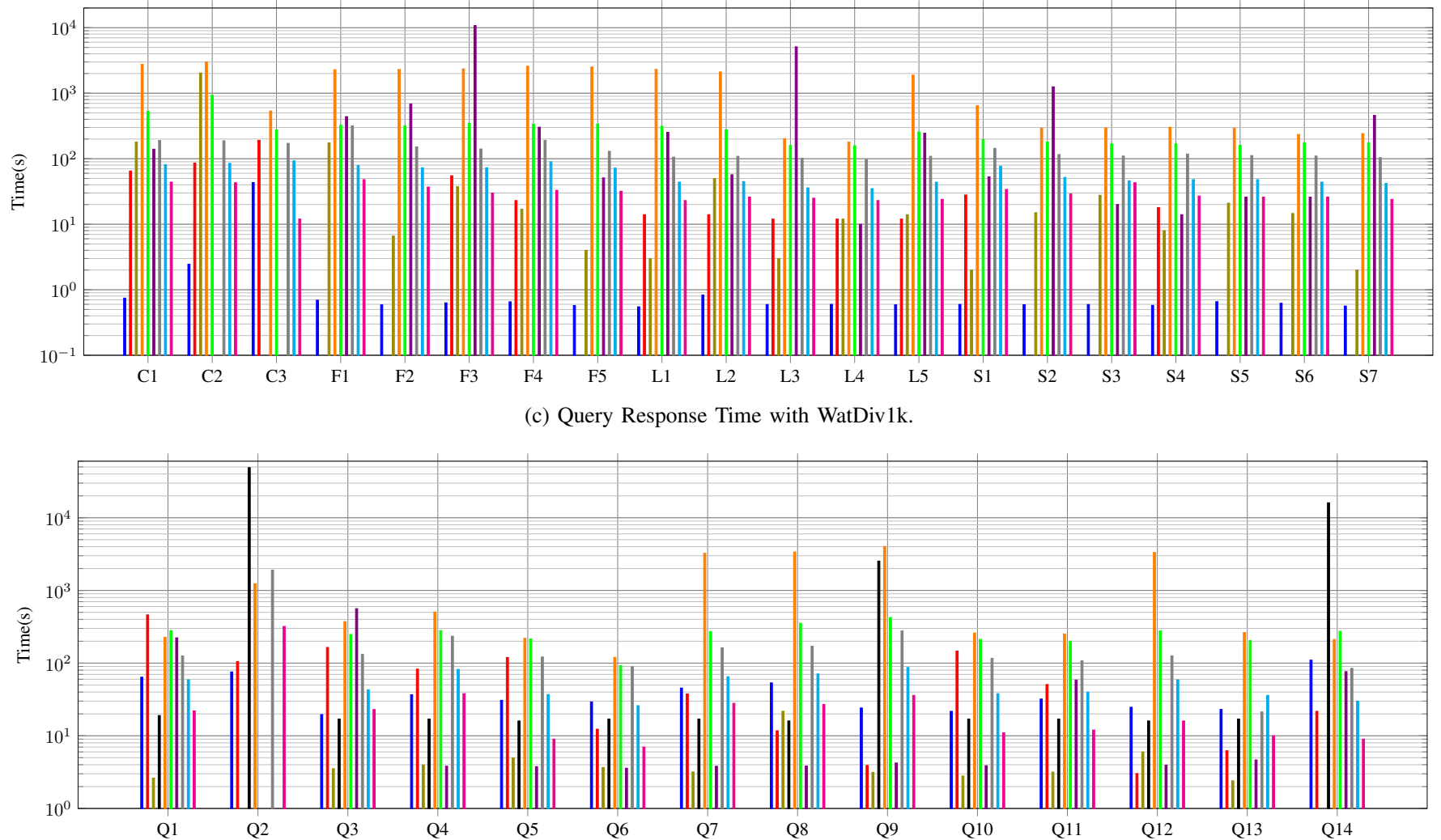

(d) Query Response Time with Lubm1k.

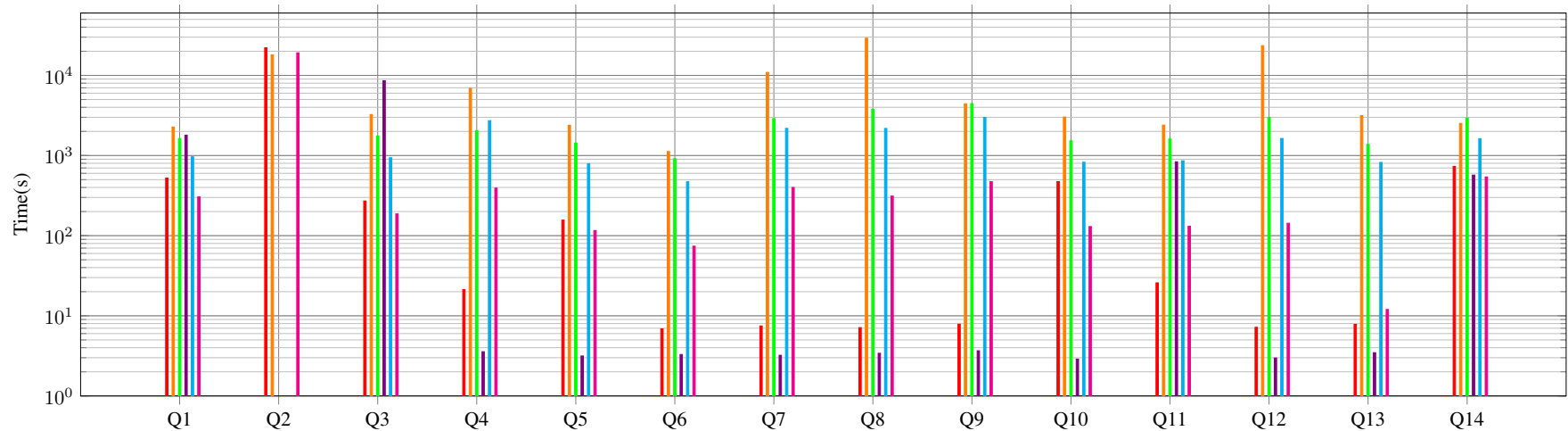

(e) Query Response Time with Lubm10k.

Fig. 1: Loading and response time with various datasets. 
4store: 4store achieves to load Lubm1k in around 3 hours (Figure 1a). But it spent nearly three days (69 hours) to ingest the 10 times larger dataset Lubm10k. While the progression was observed to be linear to load smaller datasets (i.e. a 2 times larger set was twice longer to load), 4store slowed down with a billion of triples. To execute the whole set of LUBM queries on Lubm1k (Figure 1d), 4store never spent more than one minute evaluating each query except Q1, Q2 and Q14 (respectively 64, 75 and 109 seconds). Furthermore, it achieves sub-second response time for WatDiv queries (excepting C2 and C3) with WatDiv1k (Figure 1c).

CumulusRDF: CumulusRDF is very slow to index datasets: it took almost a week only to preprocess Lubm1k (Figure 1a). By loading smaller datasets (e.g. Lubm100 or Lubm10), we notice that the empirical loading time is proportional to the dataset size. That is why we decided not to test it on Lubm10k which is 10 times larger. During the evaluation of the LUBM set of queries on Lubm1k (Figure 1d), the test of CumulusRDF revealed three points. (1) Q2 and Q9 which are the most difficult queries of the benchmark (see Section III-A) took respectively almost 5000 seconds and 2500 seconds. (2) Q14 answered in 1600 seconds seems to slow CumulusRDF because of its large output. (3) The remaining queries were all evaluated in less than 20 seconds.

CouchBaseRDF: We recall that CouchBaseRDF is an in-memory distributed datastore, which means that datasets are distributed on the main memory of the cluster's nodes. As expected, loading Lubm10k, which is larger than the whole available RAM on the cluster, was impossible. Actually, it crashed our cluster after more than 16 days i.e. all the nodes were frozen; and we had to crawl the logs in order to find that it ran out of RAM and SWAP after only indexing nearly one third of the dataset. CouchBaseRDF evaluates quickly queries on Lubm1k (Figure 1d), compared to the other evaluators; but it fails answering Q2 and Q14 throwing an exception after two minutes. We also show (Figure 1c) that CouchBaseRDF is slow to evaluate C2 (about 2000 seconds) and fails with an exception evaluating $\mathrm{C} 3$.

RYA: RYA achieves to load WatDiv1k and Lubm1k in less than one hour and preprocesses Lubm10k in less than 10 (Figure 1a). However, we note that it needs more preprocessing time with WatDiv1k (15GB) than with Lubm1k (23GB) due to the larger number of predicates WatDiv involves. RYA was not able to answer three queries: $\mathrm{C} 2 \& \mathrm{C} 3$ of WatDiv and Q2 of LUBM. In these cases, RYA runs indefinitely without failing or declaring a timeout. To answer the rest of the queries (Figures 1c \& 1d), RYA needs less than 10 seconds for most of the LUBM queries excepting Q1, Q3 and Q14. With WatDiv1k, RYA has response times varying over three orders of magnitude e.g. L4 which needs 10 seconds and F3 needs 10819. Thanks to its sorted tables (on top of Accumulo), RYA is able to answer quickly queries which involving small intermediate results; therefore, it needs the same amount of time with Lubm10k (Figure 1e) than with Lubm1k (Figure 1d).

SPARQLGX: Thanks to its data storage model (i.e. the Vertical Partitioning), SPARQLGX achieved to preprocess Lubm1k in less than one hour as it does with WatDiv1k (Figure 1a). SPARQLGX preprocesses Lubm10k in about 11 hours. As shown in Figure 1d, all queries but Q2 and Q9 have been evaluated on this dataset in less than 30 seconds.
Indeed, these two ones took respectively 250 and 36 seconds. Figure 1c shows that SPARQLGX always answer the WatDiv queries in less than one minute, and the average response time is 30 seconds.

S2RDF: While S2RDF was able to preprocess WatDiv1k and Lubm1k correctly (Figure 1a), it fails with Lubm10k throwing a memory space exception. Nonetheless, we also notice that preprocessing WatDiv1k was about two times longer than preprocessing Lubm1k; this counterintuitive observation can be explained by the vertical partitioning extension strategy used by S2RDF. Since it computes additional tables based on pre-computation of possible joins, it has to generate more additional table when the number of distinct predicate-object combinations increases. To evaluate WatDiv queries, S2RDF always needs less than 200 seconds excepting F1 (Figure 1c) and the average response time is 140 seconds. Figure 1d presents the S2RDF results with Lubm1k, we notice that all queries are aswered in less than 300 seconds excepting Q2 which exceeds one thousand seconds due to its large intermediate results that have to be shuffled across the cluster.

CliqueSquare: CliqueSquare achieves to load WatDiv1k, Lubm1k and Lubm10k (Figure 1a). Figures 1d \& 1e show how its storage model impacts its performances compared to the other evaluators. Actually, having a large number of small files allows CliqueSquare to evaluate the LUBM queries having small intermediate results in the same temporal order of magnitude on Lubm10k as the one needed on Lubm1k (see e.g. Q10). We notice that CliqueSquare cannot establish a query plan for the WatDiv queries with its SPARQL parser reporting that the URIs were not "correctly formated". We finally succeeded to evaluate some queries by modifying their syntax as explained in our website. Unfortunately, it appears that we cannot hack queries having at least such a predicate: "<...\#type $>$ " (i.e. F1, F2, F5, S2, S3, S5, S6 and S7) unless we modify Cliquesquare's source code. Nonetheless, CliqueSquare needs 12 seconds in average to answer each WatDiv linear query, and spends more than one minute to evaluate each complex one (Figure 1c).

PigSPARQL: PigSPARQL evaluates directly the queries after a translation from SPARQL to a PigLatin sequence. Thus, there is no preprocessing phase, we just have to copy the triple file on the HDFS. As shown in Figure 1d, PigSPARQL needs more than one thousand seconds to answer queries 2, 7 , 8, 9 and 12 on Lubm1k while the other queries take around 200 seconds. We observe the same behaviors when evaluating these queries on Lubm10k (Figure 1e). Similarly, the same order of magnitude applies with WatDiv1k (Figure 1c).

RDFHive: RDFHive only needs a triple file loaded on the HDFS to start evaluating queries. It appears that RDFHive was unable to answer Q2 of LUBM i.e. no matter the time allowed, it could not finish the evaluation. On Lubm1k (Figure 1d), we also notice that each remaining query is evaluated on Lubm $1 \mathrm{k}$ in a 200 to 450 seconds period with a 256 -second average response time. Similarly (Figure 1c), RDFHive has 289-second average response time with WatDiv1k.

SDE: Since SDE is a SPARQL direct evaluator, it does not need any preprocessing time to ingest datasets. Its average response times with WatDiv1k, Lubm1k and Lubm10k (Figures 1c, 1d \& 1e) are respectively 60, 51 and 1460 seconds. 
We observe that the average response time with Lubm10k is about 28 times larger than the one with Lubm1k (which is 10 times larger) indeed Q4, Q7, Q8, Q9, Q12 and Q14 do not perform well because of their large intermediate results.

General Observations: A first lesson learned is that, for the same query on the same dataset, elapsed times can differ very significantly (the time scale being logarithmic) from one system to another (as shown for instance on Figure 1d).

Interestingly, we also observe that, even with large datasets, most queries are not harmful per se, i.e. queries that incur long running times with some implementations still remain in the "comfort zone" for other implementations, and sometimes even representing a case of demonstration of efficiency for others. For example, the response times for Q12 with Lubm1k (see Figure 1d) span more than 3 orders of magnitude. Interestingly and more generally, for each query, there is at least a difference of one order of magnitude between the times spent by the fastest and the slowest evaluators.

These observations gave rise to the further comparative analysis guided by criteria (and supplemented with additional metrics) that we present in Section V.

\section{Comparative Analysis Driven By Features}

The variety of RDF application workloads makes it hard to capture how well a particular system is suited compared to the others in a way based exclusively on time measurements. For instance, consider these five features that have different needs and where the main emerging requirement is not the same:

- Velocity: applications might favour the fastest possible answers (even if that means storing the whole dataset in RAM, when possible).

- Immediacy: applications might need to evaluate some SPARQL queries only once. This is typically the case of some pipeline extraction applications that have to extract data cleaned only once.

- Dynamicity: applications might need to deal with dynamic data, requiring to react to frequent data updates. In this case a small preprocessing time (or the capacity to react to updates in an incremental manner) is important.

- Parsimony: applications might need to execute queries while minimizing some of the resources, even at the cost of slower answers. This is for example the case of background batch jobs executed on cloud services where the main factors for the pricing of the service are network, CPU and RAM usage.

- Resiliency: applications that process very large data sets (spanning accross many machines) with complex queries (taking e.g. days to complete) might favour forms of resiliency for trying to avoid as much as possible to recompute everything when a machine fails because it is likely to happen.

Since many applications actually combine these requirements by affecting more or less importance to each, we believe that they represent a good basis on which to compare the tested systems. In this Section, we thus further compare the tested stores by analysing the metrics introduced in Section III-B according to the five aforementioned requirements. For the sake of brevity, we will directly refer to these requirements as "velocity", "immediacy", "dynamicity", "parsimony" and "resiliency" in the rest of the paper.

\section{A. Velocity The Faster, The Better}

Figure 1d shows the time per query using Lubm1k as dataset for each tested store. The logarithmic scale allows to easily observe the various magnitude orders required to execute queries. It is then possible to notice significant differences between e.g. CumulusRDF that needs more than $10^{4}$ seconds to answer Q2 or Q14 while for instance 4store always has response times included in $[10,100]$ seconds. More generally, it appears that Q2 incurs the longest response times because of its triangular pattern and its large intermediate results. If we compute the sum of the response times for all the queries of Lubm1k for each evaluator, we notice that our candidates have performances spanning over three orders of magnitude from 568 seconds with SPARQLGX and 67718 seconds with CumulusRDF. Thereby, to execute the whole set of 14 LUBM queries, SPARQLGX and 4store constitute the fastest solutions.

\section{B. Immediacy Preprocessing is Investing}

The preprocessing time required before querying can be seen as an investment i.e. taking time to preprocess data (load/index) should imply faster query response time, offseting the time spent in preprocessing. To illustrate when the tradeoff is really worth, Figure 2 presents the preprocessing costs for Lubm1k and WatDiv1k in various cases. In other words, we draw on a logarithmic time scale for each evaluator the affine line $y=a x+b$ where $a$ is the average time required to evaluate one of the considered queries and where $b$ is the preprocessing time; for instance in Figure 2c, $a$ will represent the average time to evaluate one WatDiv linear query.

Among competitors, we distinguish the set of "direct evaluators" (See Table I) that are capable of evaluating SPARQL queries at no preprocessing cost (they do not require any preprocessing of RDF data): PigSPARQL, RDFHive and SDE. As shown in Figure 2, SDE outperforms all the other datastores if less than 20 queries are evaluated. Beyond this threshold, SPARQLGX or RYA become more interesting. In addition, we also notice that in some cases (for instance Q8, see Figure 2b) PigSPARQL provide worse performances than RYA or SPARQLGX all the time.

These statements are also related to RDF storage approaches; indeed, the more complex it is, the less immediacyefficient the evaluator is. As a consequence, we can rank for this feature the various storage methods from the best ones: first the schema-carfree triple table of the direct evaluators, next the vertical partitioning, then the key-value table (e.g. RYA) and finally the complicated indexing methods.

\section{Dynamicity Changing Data}

We now examine how the tested stores can be set up to react to frequent data changes. The $\mathrm{W} 3 \mathrm{C}$ proposes an extension of SPARQL to deal with updates ${ }^{6}$. Instead of re-loading all the

\footnotetext{
${ }^{6}$ https://www.w3.org/Submission/SPARQL-Update/
} 


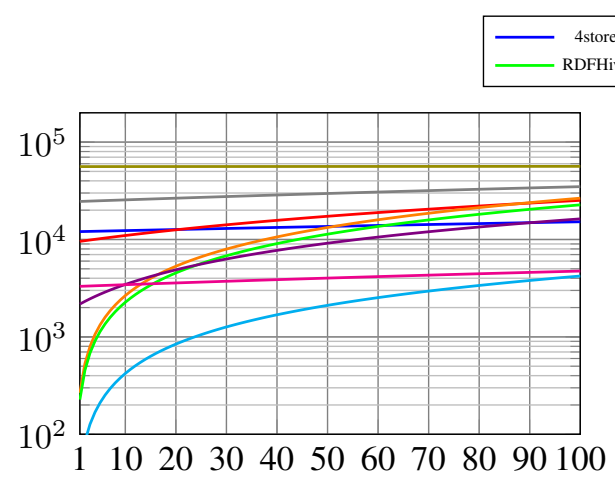

(a) lubm1k: Q1,Q3,Q5,Q10,Q11,Q13

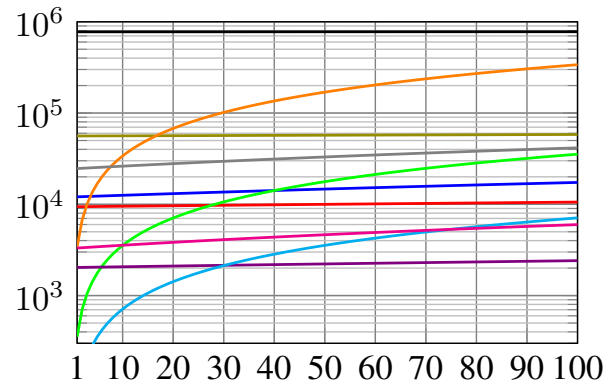

(b) lubm1k: Q8

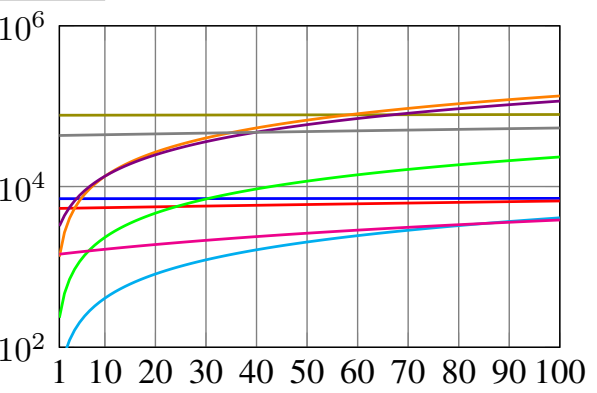

(c) watdiv1k: L1,L2,L3,L4,L5

Fig. 2: Tradeoff between preprocessing and query evaluation times (seconds).

datasets after each single change, some solutions can be set up to load bulks of updates. To the best of our knowledge, there is no widely-used benchmark dealing exclusively with the SPARQL Update extension. That is why we develop a basic experimental protocol based on both LUBM and WatDiv benchmarks. It can be divided into three steps: (1) We load a large dataset i.e. Lubm1k (Table II) and evaluate the simple LUBM query Q1 then we measure performances for preprocessing and query evaluation. (2) We add a few RDF triples to modify the output of Q1; we run again Q1 and then remove the freshly added triples while measuring the time for each step. (3) Finally, we reproduce the previous step with a larger number of triples using WatDiv1 (which contains about one hundred thousand triples) and querying with $\mathrm{C} 1$. Although simple, our protocol allows testing the several features such as inserting/deleting a few triples and a large bulk of triples. The benchmarked datastores exhibit various behaviors. First, the direct evaluators (e.g. PigSPARQL, RDFHive and SDE) evaluate queries without requiring a preprocessing phase. In that case, updating a dataset boils down to editing a file on the HDFS and retriggering query evaluation. Second, other datastores simply do not implement any support (even partial) of updates. This category of stores (e.g. S2RDF, CumulusRDF, CouchBaseRDF, RYA or CliqueSquare) thus forces the reprocessing of the whole dataset. Third, some of the benchmarked datastores are able to deal with dynamic datasets i.e. 4store and SPARQLGX. 4store implements the SPARQL Update extension whereas SPARQLGX offers a set of primitives to add or delete sets of triples. Moreover, unlike 4store, SPARQLGX is also able to delete in one action a large set of triples, whereas 4 store needs to execute several "Delete Data"-processes if the considered set cannot fit in memory.

\section{Parsimony Share and Parallelize}

Figure 3 shows how each cluster node behaves during the Lubm1k query phase and thus provides an idea of how the evaluators allocate resources across the cluster. Such a visualization also confirms some properties one can guess about evaluators. For example by observing the 4store CPU average usage in Figure 3a, we can highlight its storage architecture: the Nodes 6 to 10 are more CPU-active during the process (about $40 \%$ of CPU whereas other nodes use about $20 \%$ ) and thus correspond to the 4 store computing nodes while the other ones (excepting the driver on Node1) correspond to the 4store storing nodes. In addition, the number of bytes sent across the network provides clues to identify the evaluator driver nodes (Figure 3b) i.e. it appears that the Node1 of 4store and RDFHive sends at least 10 times more data than the other nodes (which are receiving). According to several observations made previously (see e.g. Section IV), we know that the RAM usage can be a bottleneck for SPARQL evaluation. Representing in Figure 3c the maximum allocated RAM per node during the Lubm1k query phase, we observe that several evaluators are closed to the maximum possible of $16 \mathrm{~GB}$ per node (see Section III-C): CouchBaseRDF which is an inmemory datastore, CumulusRDF and the three Spark-based evaluators e.g. S2RDF, SDE and SPARQLGX. On the other hand, 4store and CliqueSquare need in average less than one order of magnitude than it is possible to allocated while being temporally efficient (see e.g. Section V-A).

Figure 4 presents resource usages correlated with Lubm1k query evaluation. We give three curves for each evaluator during the Lubm1k query phase: first, the network traffic (sent and received bytes); second, the disk activity (read and write bytes); third, the CPU usage. Moreover, we also divide the time dimension according the needed response times of LUBM queries to observe the resource consumption during one designated query at a glance. We observe that Network and Disk peaks are often synchronous, which means the evaluator reads and transmits or receives and saves data. These correlations are especially observed with the direct evaluators since they have to read at least once the whole dataset to evaluate a SPARQL query and also have to shuffle intermediate results to join them (see e.g. Figures $4 \mathrm{c}, 4 \mathrm{f} \& 4 \mathrm{i}$ ). In addition, we also remark that thanks to their storage models, 4store CliqueSquare or CouchBaseRDF never have to read large amounts of data and we can only observe network peaks when the query has large intermediate results or outputs such as Q14 for example (see e.g. Figures $4 \mathrm{a}, 4 \mathrm{~d} \& 4 \mathrm{~h}$ ).

Paying attention to resource consumption thereby provides information on the real evaluator behaviors. Actually, we found that some systems that dominate in previous features (e.g SDE for Immediacy) are in fact costly for the cluster in terms of RAM allocation of CPU average usage. Moreover, we also highlight that the Spark-based evaluators have a selfish 


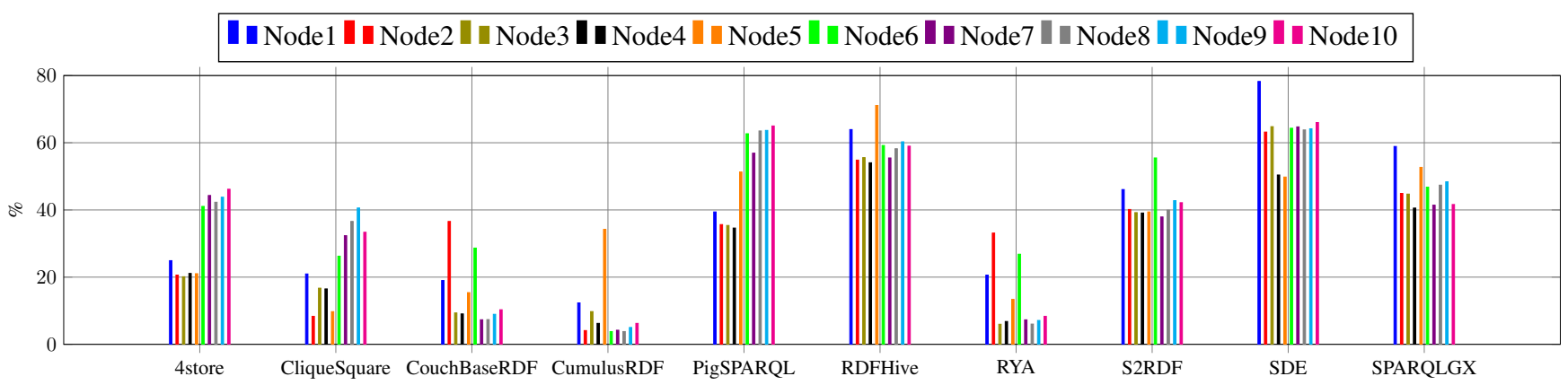

(a) Average CPU.

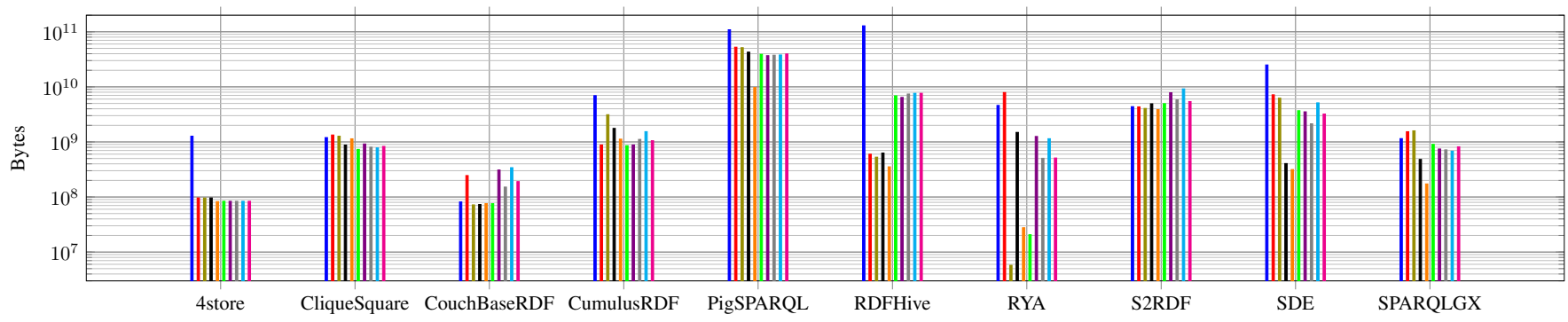

(b) Total bytes sent.

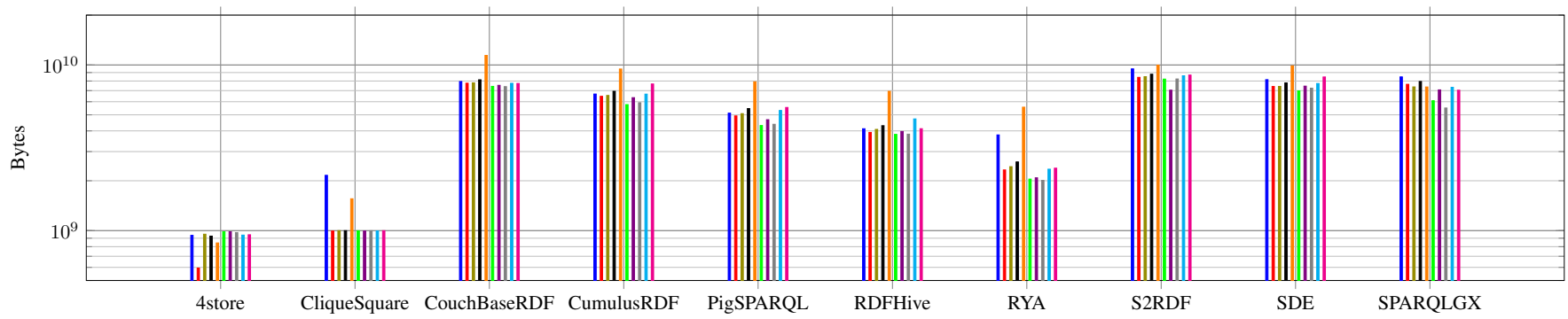

(c) Maximum allocated RAM.

Fig. 3: CPU, Network and RAM consumptions per node during Lubm1k query phase.

behavior by using as much resources as possible in order to provide an answer as quickly as possible. As a conclusion, if one needs to run concurrent processes while evaluating SPARQL queries (e.g. running a SQL service or data processing pipelines at the same time), one should rather prefer evaluators whose data storage models are optimized such as 4store or CliqueSquare.

\section{E. Resiliency Having Duplicates}

Data Resiliency: When an application processes a very large dataset stored across many machines, it is interesting for the system to implement some level of tolerance in case a datanode is lost. To implement data resilience, stores typically replicate data across the cluster which implies a larger disk footprint. For our experiments, we stick to the default replication parameters. As a consequence, the HDFS-based systems have their data replicated twice and provide some level of data resilience. Table III presents the effective disk footprints (including replication) with Lubm1k and WatDiv1k where the HDFS-based systems are outlined in gray. Due to their preprocessing methods, we note that S2RDF and CliqueSquare need

\begin{tabular}{|c|c|c|}
\hline Systems & Lubm1k (GB) & WatDiv1k (GB) \\
\hline S2RDF & $\mathbf{1 3 . 0 5 7}$ & 15.150 \\
RYA & 16.275 & $\mathbf{1 1 . 0 2 7}$ \\
CumulusRDF & 20.325 & - \\
4store & 20.551 & 14.390 \\
CouchBaseRDF & 37.941 & 20.559 \\
SPARQLGX & 39.057 & 23.629 \\
CliqueSquare & 55.753 & 90.608 \\
PigSPARQL & 72.044 & 46.797 \\
RDFHive & 72.044 & 46.797 \\
SDE & 72.044 & 46.797 \\
\hline
\end{tabular}

TABLE III: Disk Footprints (including replication).

more disk space to store WatDiv1k than Lubm1k whereas this last one is larger (see Table II). Furthermore, counterintuitively, it appears that evaluators having replicated data can have lighter disk footprints than not-replicated ones e.g S2RDF and RYA versus CouchBaseRDF.

Computation Resiliency: If an application has to evaluate complex queries (taking e.g. days), it is interesting for the system not to be forced to compute everything from scratch 


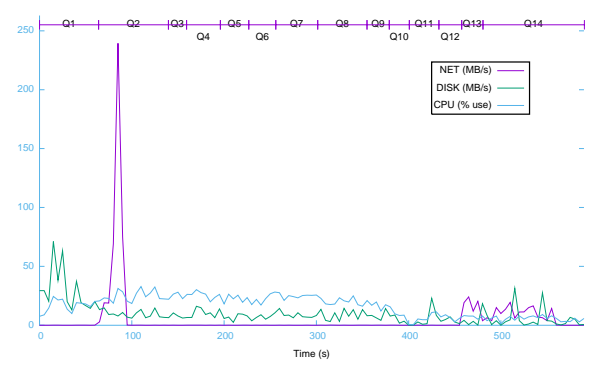

(a) 4store

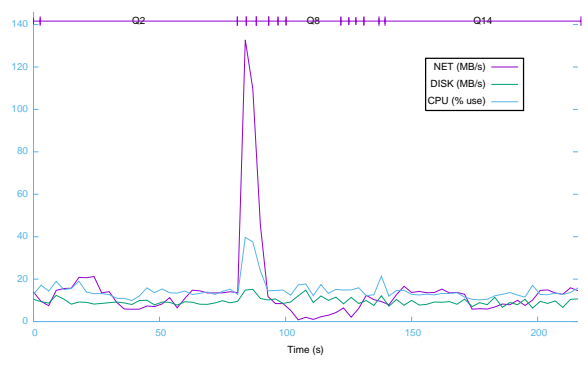

(d) CouchBaseRDF

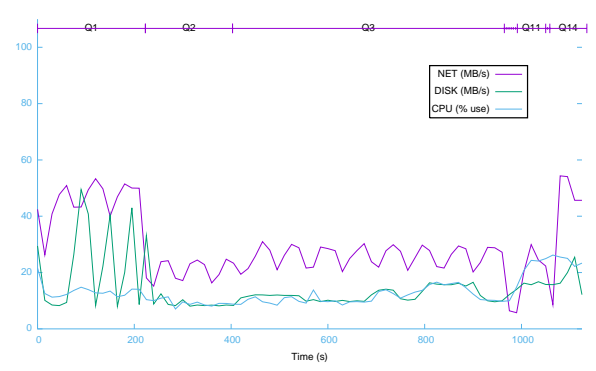

(g) RYA

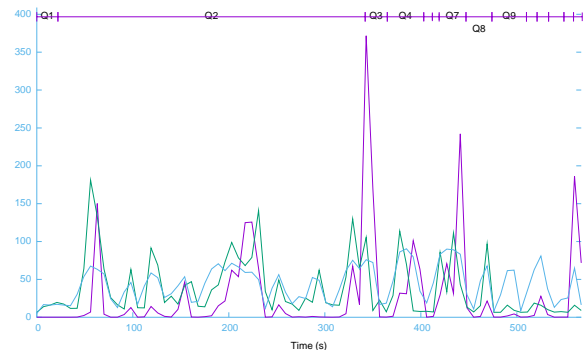

(b) SPARQLGX

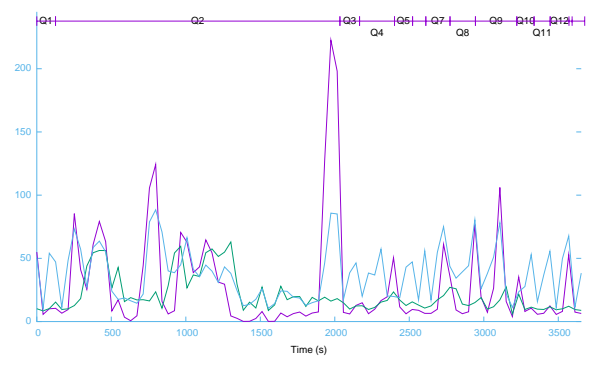

(e) S2RDF

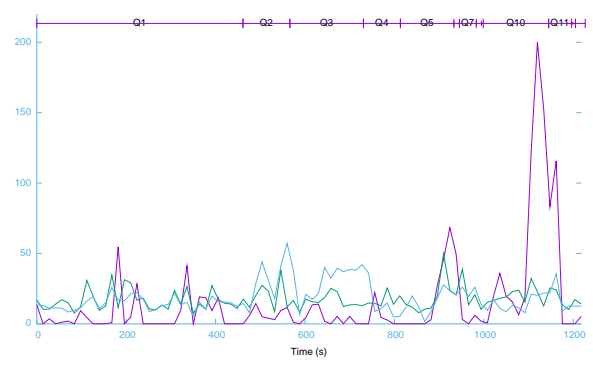

(h) CliqueSquare

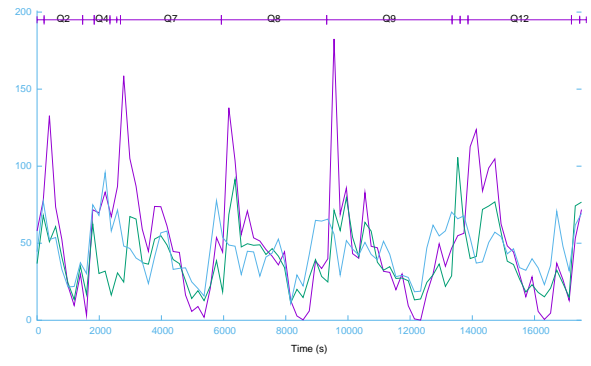

(c) PigSPARQL

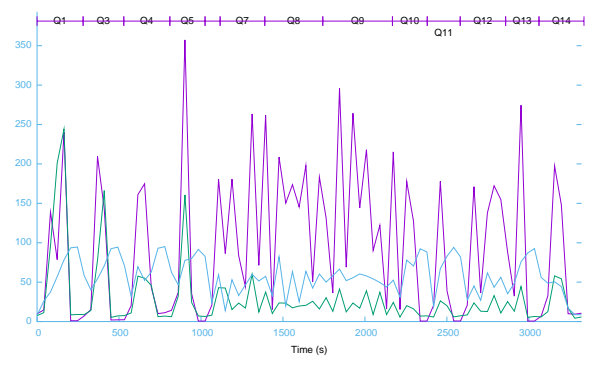

(f) RDFHive

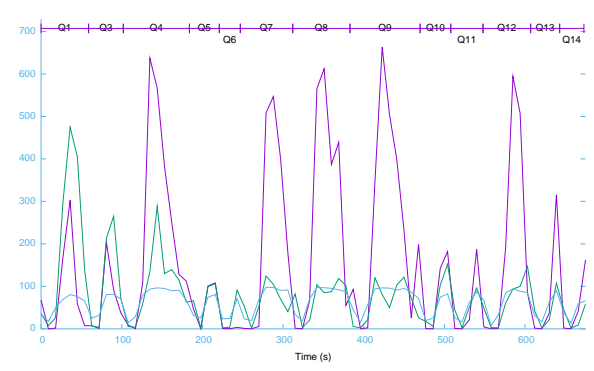

(i) $\mathrm{SDE}$

Fig. 4: Resource consumption during Lubm1k query phase.

whenever a machine becomes unreachable. This situation is likely to happen for a variety of reasons (e.g. reboot, failure, network latency). The tested systems exhibit several behaviours when a machine fails during computation. For stores having no data replication, the loss of any machine can stop the computation if the lost data fragment is mandatory; thus some stores fail when a machine is lost: 4store and $\mathrm{Cu}$ mulusRDF; whereas CouchBaseRDF adopts another method waiting seven minutes until the return of the machine. More generally, the HDFS-based triplestores cannot lose mandatory fragments of data, thereby RDFHive, SPARQLGX, SDE, RYA, and CliqueSquare still succeed when one (or even two) machine fails during computation; however, PigSPARQL waits indefinitely the return of the lost partition. For stores having a master/slave structure e.g. SPARQLGX, the loss of the node hosting the master process prevents any result to be obtained. From our tests, only two different methods successfully faced a loss of worker nodes: (1) waiting for their returns e.g. CouchBaseRDF and PigSPARQL; (2) using the remaining nodes and benefiting from data replication e.g. CliqueSquare, RDFHive, RYA, S2RDF, SDE, SPARQLGX.

\section{F. Summary At a glance}

Figure 5 presents a Kiviat chart in which the tested systems are ranked, based on Lubm $1 \mathrm{k}$ and WatDiv $1 \mathrm{k}$ according to all the features already discussed in Section V. More particularly, evaluator ranks on the two "velocity" axes (one for Lubm1k and one for WatDiv1k) are based on average response time considering only successful queries. This representation gives at a glance clues to select an evaluator. For instance it appears that 4store is especially relevant when velocity and parsimony are important and less importance is given to resiliency. SDE also appears as a reasonnable choice when all criteria (including its potential cost on a cloud platform) but parsimony matter.

\section{RELATED WORK}

This study benefited from the extensive earlier works on benchmarks for RDF systems. There are many benchmarks designed for evaluating RDF systems [18]-[25]. Some of them are particularly popular: LUBM [19], WatDiv [20], $\mathrm{SP}^{2}$ Bench [25], DBpedia Bench [23], BSBM [24], and RBench [18]. We notably reused LUBM [19] and WatDiv [20] 


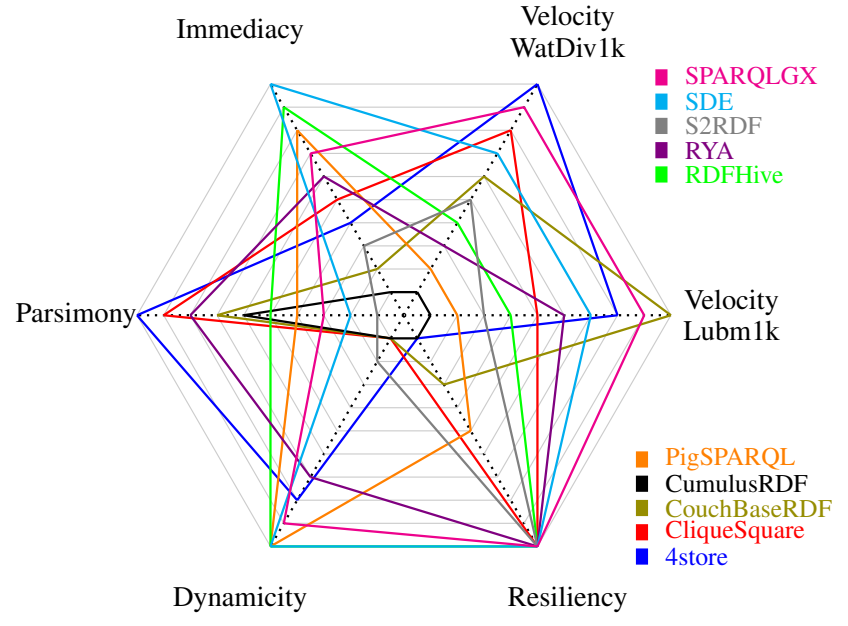

Fig. 5: System Ranking (farthest is better).

for testing the BGP fragment, and because we wanted deterministic data generators for ensuring reproducibility of our results. Compared to all these works, we focus on testing distribution techniques by considering a set of 10 state-of-theart implementations; see e.g. [3], [14], [26] for recent surveys about distributed RDF datastores and their storage approaches. Compared to studies included in the aforementioned benchmarks, we consider more competing implementations on a common ground. Furthermore, while earlier works on RDF benchmarks exclusively focused on measuring elapsed times (and sometimes disk footprints), we measure a broader set of indicators encompassing e.g. network usage. This allows to refine the comparative analysis according to features and requirements from a slightly higher perspective, as discussed in Section V. This also allows to more precisely identify the bottlenecks of each system when they are pushed to the limits.

Finally, this work was inspired by the empirical study carried out by Cudré $e t$ al. where five distributed RDF datastores using various NoSQL backends were evaluated [9]. Our work does not invalidate earlier results but supplement them with more results. In particular, in the present work, we update the list of evaluators (we consider more of them, with more recent ones) and we also focus on ranking the candidates depending on various features thanks to the broader set of metrics we analysed.

\section{CONCLUSION}

We conducted an empirical evaluation of 10 state-of-theart distributed SPARQL evaluators on a common basis. By considering a full set of metrics, we improve on traditional empirical studies which usually focus exclusively on temporal considerations. We proposed five new dimensions of comparison that help in clarifying the limitations and advantages of SPARQL evaluators according to use cases with different requirements.

\section{REFERENCES}

[1] P. Hayes and B. McBride, "RDF semantics," W3C Rec., 2004.

[2] "SPARQL 1.1 overview," March 2013, http://www.w3.org/TR/sparq111overview/.
[3] Z. Kaoudi and I. Manolescu, "RDF in the clouds: a survey," The VLDB Journal, vol. 24, no. 1, pp. 67-91, 2015.

[4] J. Dean and S. Ghemawat, "Mapreduce: simplified data processing on large clusters," Communications of the ACM, vol. 51, no. 1, pp. 107113,2008

[5] M. Zaharia, M. Chowdhury, T. Das, A. Dave, J. Ma, M. McCauley, M. J. Franklin, S. Shenker, and I. Stoica, "Resilient distributed datasets: A fault-tolerant abstraction for in-memory cluster computing," NSDI, 2012.

[6] C. Olston, B. Reed, U. Srivastava, R. Kumar, and A. Tomkins, "Pig latin: a not-so-foreign language for data processing," in SIGMOD. ACM, 2008, pp. 1099-1110.

[7] S. Harris, N. Lamb, and N. Shadbolt, "4store: The design and implementation of a clustered RDF store," SSWS, 2009.

[8] A. Schätzle, M. Przyjaciel-Zablocki, S. Skilevic, and G. Lausen, "S2RDF: RDF querying with SPARQL on spark," $V L D B$, pp. 804-815, 2016.

[9] P. Cudré-Mauroux, I. Enchev, S. Fundatureanu, P. Groth, A. Haque, A. Harth, F. L. Keppmann, D. Miranker, J. F. Sequeda, and M. Wylot, "NoSQL databases for RDF: An empirical evaluation," ISWC, pp. 310325, 2013.

[10] Abadi, Marcus, Madden, and Hollenbach, "Scalable semantic web data management using vertical partitioning," $V L D B, 2007$.

[11] R. Punnoose, A. Crainiceanu, and D. Rapp, "RYA: a scalable RDF triple store for the clouds," in International Workshop on Cloud Intelligence. ACM, 2012, p. 4.

[12] K. Shvachko, H. Kuang, S. Radia, and R. Chansler, "The hadoop distributed file system," in Mass Storage Systems and Technologies (MSST), 2010 IEEE 26th Symposium on. IEEE, 2010, pp. 1-10.

[13] F. Goasdoué, Z. Kaoudi, I. Manolescu, J.-A. Quiané-Ruiz, and S. Zampetakis, "Cliquesquare: Flat plans for massively parallel RDF queries," in ICDE. IEEE, 2015, pp. 771-782.

[14] D. C. Faye, O. Curé, and G. Blin, "A survey of RDF storage approaches," Arima Journal, vol. 15, pp. 11-35, 2012.

[15] G. Ladwig and A. Harth, "CumulusRDF: linked data management on nested key-value stores," SSWS 2011, p. 30, 2011.

[16] D. Graux, L. Jachiet, P. Genevès, and N. Layaïda, "SPARQLGX: Efficient Distributed Evaluation of SPARQL with Apache Spark," ISWC, 2016.

[17] A. Schätzle, M. Przyjaciel-Zablocki, and G. Lausen, "PigSPARQL: Mapping SPARQL to pig latin," in Proceedings of the International Workshop on Semantic Web Information Management. ACM, 2011, p. 4.

[18] S. Qiao and Z. M. Özsoyoğlu, "Rbench: Application-specific RDF benchmarking," in SIGMOD. ACM, 2015, pp. 1825-1838.

[19] Y. Guo, Z. Pan, and J. Heflin, "LUBM: A benchmark for OWL knowledge base systems," Web Semantics, 2005.

[20] G. Aluç, O. Hartig, M. T. Özsu, and K. Daudjee, "Diversified stress testing of RDF data management systems," in ISWC. Springer, 2014, pp. 197-212.

[21] R. Angles, P. Boncz, J. Larriba-Pey, I. Fundulaki, T. Neumann, O. Erling, P. Neubauer, N. Martinez-Bazan, V. Kotsev, and I. Toma, "The linked data benchmark council: a graph and RDF industry benchmarking effort," ACM SIGMOD Record, vol. 43, no. 1, pp. 27-31, 2014.

[22] G. Demartini, I. Enchev, M. Wylot, J. Gapany, and P. Cudré-Mauroux, "Bowlognabench - Benchmarking RDF Analytics," in International Symposium on Data-Driven Process Discovery and Analysis. Springer, 2011, pp. 82-102.

[23] M. Morsey, J. Lehmann, S. Auer, and A.-C. N. Ngomo, "DBpedia SPARQL Benchmark - Performance assessment with real queries on real data," ISWC, pp. 454-469, 2011.

[24] C. Bizer and A. Schultz, "The berlin SPARQL benchmark," IJSWIS, 2009.

[25] M. Schmidt, T. Hornung, G. Lausen, and C. Pinkel, "SP${ }^{2}$ Bench: a SPARQL performance benchmark," ICDE, pp. 222-233, 2009.

[26] G. A. Atemezing and F. Amardeilh, "Benchmarking commercial rdf stores with publications office dataset," in European Semantic Web Conference. Springer, 2018, pp. 379-394. 